\title{
How general is anomeric retention during collision-induced dissocia- tion of glycans?
}

\author{
Robert P. Pellegrinelli, Lei Yue, Eduardo Carrascosa, Stephan Warnke, Ahmed Ben Faleh, and Thomas \\ R. Rizzo* \\ Laboratoire de Chimie Physique Moléculaire, Ecole Polytechnique Fédérale de Lausanne, EPFL SB ISIC LCPM, Station 6, \\ CH-1015 Lausanne, Switzerland.
}

\begin{abstract}
Despite the essential role that glycans play in many biological processes, their isomeric complexity makes their structural determination particularly challenging. Tandem mass spectrometry has played a central role in glycan analysis, and recent work has shown that fragments generated by collision-induced dissociation (CID) of disaccharides can retain the anomeric configuration of the glycosidic bond. If this result proves to be general, it would provide a powerful new tool for glycan sequencing. In this work, we use messenger-tagging infrared (IR) spectroscopy to investigate the generality of anomer retention in CID by exploring different fragmentation channels in glycans of increasing complexity. Our results demonstrate that anomericity seems to be retained irrespective of fragment size and branching.
\end{abstract}

Understanding the structure of glycans is of crucial importance, since glycoproteins at the surface of most living cells influence cellular interaction with external entities. ${ }^{1-}$

${ }^{3}$ The isomeric complexity of glycans, together with the fact that their synthesis is not template-driven, makes it difficult to determine their primary structure by the standard techniques used for DNA and protein sequencing. Determining the anomericity of the glycosidic bond stands out as a particular challenge. Very recently, Compagnon and coworkers showed spectroscopic evidence that $C_{1}$ fragments (Scheme 1) generated by collision-induced dissociation (CID) of lithiated disaccharides retain the anomeric configuration of the glycosidic bond, ${ }^{4}$ and this has also been investigated by coupling ion mobility with tandem MS. ${ }^{5-6}$ This finding raises many questions: How general is anomeric retention in the gas phase? Does it apply to larger, more complex glycans? Does it apply to larger fragments? The answers to these questions are likely to have profound implications for glycan analysis.

The anomericity of the glycosidic bond, which can exist in either the $\alpha$ or $\beta$ configuration, is commonly determined by coupling exoglycosidase digestion with liquid chromatography. ${ }^{7-10}$ However, this usually involves lengthy incubation times and multiple chromatographic separations. Techniques such as $\mathrm{NMR}^{11}$ and $\mathrm{X}$-ray crystallography $^{12}$ can provide detailed structural information including the anomeric configuration, but they require a relatively large amount of sample, which is often not available in the case of glycans. Tandem mass spectrometry $\left(\mathrm{MS}^{\mathrm{n}}\right)$ has the advantage of providing rapid structural information while requiring small amounts of sample. Branching, bond position, and anomericity can be successfully determined by analyzing the fragments from relatively small glycans, ${ }^{13-20}$ although the latter requires observing cross-ring fragments that preserve the anomeric configuration of the glycosidic bond. ${ }^{10,21-22}$ Nevertheless, tandem MS is typically unable to fully distinguish between all isomeric forms.

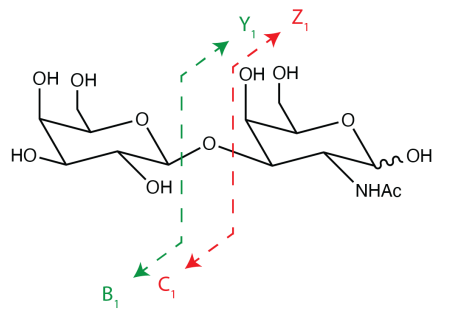

Scheme 1 - Nomenclature for $\mathrm{B} / \mathrm{Y}$ and $\mathrm{C} / \mathrm{Z}$ fragments of glycans. ${ }^{23}$ It should be noted that both the $\mathrm{C}$ and $\mathrm{Y}$ fragments carry a hydrogen atom to form an intact glycan.

Infrared (IR) spectroscopy is a promising tool for glycan analysis, as the vibrational spectrum is extremely sensitive to the slightest of structural differences. Recently, room-temperature infrared multiphoton dissociation (IRMPD) has been used to fingerprint monosaccharide fragments, ${ }^{4}$ while cryogenic IR spectroscopy has been used by Pagel and coworkers ${ }^{24}$ as well as our group ${ }^{25-26}$ to identify spectral fingerprints of increasingly complex sets of isomeric glycans. Cryogenic spectroscopy has the advantage of eliminating thermal inhomogeneous broadening, resulting in significantly increased resolution for large molecules with congested vibrational spectra. ${ }^{27}$ In this work, we use cryogenic messenger-tagging infrared spectroscopy and ultrahigh-resolution ion mobility spectrometry (IMS) to investigate the generality of anomeric retention upon CID. 
A detailed description of the instrument used in this study can be found elsewhere. ${ }^{28}$ Briefly, singly sodiated glycans are produced in the gas phase by nano-electrospray ionization (nESI), radially confined in an ion funnel, and accumulated in a hexapole ion trap. Fragmentation is induced by collisions in the hexapole at a pressure of $10^{-1}$ mbar by accelerating the ions through a potential difference of $\sim 200 \mathrm{~V}$ between the funnel exit and hexapole bias. Fragments of a specific $\mathrm{m} / \mathrm{z}$ are selected by a first quadrupole mass filter and sent to an octupole ion trap enclosed in a copper housing and maintained at $60 \mathrm{~K}$, where they are cooled upon collisions with cold helium and complexed with a weakly bound nitrogen molecule. Nitrogen-tagged ions are irradiated every other trapping cycle by a single IR pulse from an optical parametric oscillator (OPO) before being sent through a second quadrupole mass filter and detected by a channeltron.

In the event of a resonant IR transition in the irradiated ion, the absorbed energy is intramolecularly redistributed, leading to the detachment of the weakly bound nitrogen tag. The IR spectrum is obtained by monitoring the depletion of the signal at the tagged-ion mass as a function of IR wavelength in a laser-on/laser-off experiment.

We first tested anomeric retention in the $\mathrm{C}_{1}$ fragments of Gal $\alpha(1-3)$ GalNAc and Gal $\beta(1-3)$ GalNAc by comparing their cryogenic vibrational spectra (Figs. 1(a, d) with those of the methylated $\alpha$ and $\beta$ anomers of galactose (Figs. 1(b, c)).

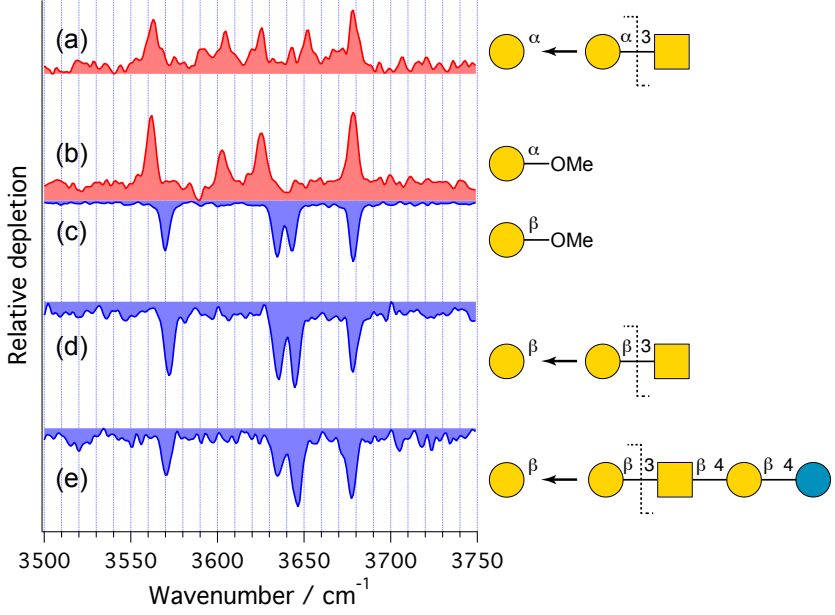

Figure 1: Cryogenic vibrational spectra of the $C_{1}$ fragments of galactose-containing disaccharides $(\mathrm{a}, \mathrm{d})$ and of a tetrasaccharide (e) and their comparison to the methylated anomers of galactose $(b, c)$.

Because methylated galactose does not interconvert at the anomeric carbon, the spectra of the substituted monosaccharides should provide a good model of anomerically pure galactose, except for the absence of the anomeric $\mathrm{OH}$ stretch band, which occurs at $3652.5 \mathrm{~cm}^{-1}$ in the $\alpha$ anomer and $3644.5 \mathrm{~cm}^{-1}$ in the $\beta$ anomer. Figure 1(e) shows the IR spectrum of the $C_{1}$ fragment generated from a tetrasaccharide Gal $\beta(1-3)$ GalNAc $\beta(1-4)$ Gal $\beta(1-4)$ Glc. All spectra of the $C_{1}$ fragment, which is itself an intact galactose, show a clear correspondence with those of the respective methylated galactose anomer. This demonstrates that $\mathrm{C}_{1}$ fragments produced from an $\alpha$ glycosidic linkage represent the anomerically pure $\alpha$ monosaccharide, and that fragmentation of the $\beta$ glycosidic linkage gives the anomerically pure $\beta$ monosaccharide, irrespective of the size of the initial glycan.

To test whether anomer retention also occurs in N-acetylated C-fragments of glycans, we performed similar experiments on two disaccharides and a trisaccharide containing GalNAc at the non-reducing end. Figure 2 compares spectra of $\mathrm{C}_{1}$ fragments to the isomer-specific spectra of the monosaccharide GalNAc obtained using an apparatus that employs ultrahigh-resolution IMS based on structures for lossless ion manipulations (SLIM) ${ }^{29-30}$ to separate the two anomers before measuring their spectra. ${ }^{31}$ Two ion-mobility peaks for GalNAc were resolved after 11 cycles on the SLIM board, which represents a total drift path of $\sim 20 \mathrm{~m}$. The vibrational spectrum of the ions contained in the first mobility peak (Fig. 2(c)) matches that of the $\mathrm{C}$ fragments generated from an $\alpha$ glycosidic linkage (Figs. 2(a, b)), while the spectrum of the ions in the second mobility peak Fig. 2(e) matches the fragment generated from a $\beta$ glycosidic linkage (Fig. 2(d)). It should be noted that because the two mobility peaks were not baseline separated, vibrational bands from the major component (Fig. 2(c)) appear in the spectrum of the minor component (Fig. 2(d)), but with reduced intensity. However, one can see that the peak at $3465 \mathrm{~cm}^{-1}$, for example, is absent from the spectrum of Fig. 2(e), suggesting that the GalNAc fragment from GalNAc $\beta(1-$ 3) Gal is anomerically pure.

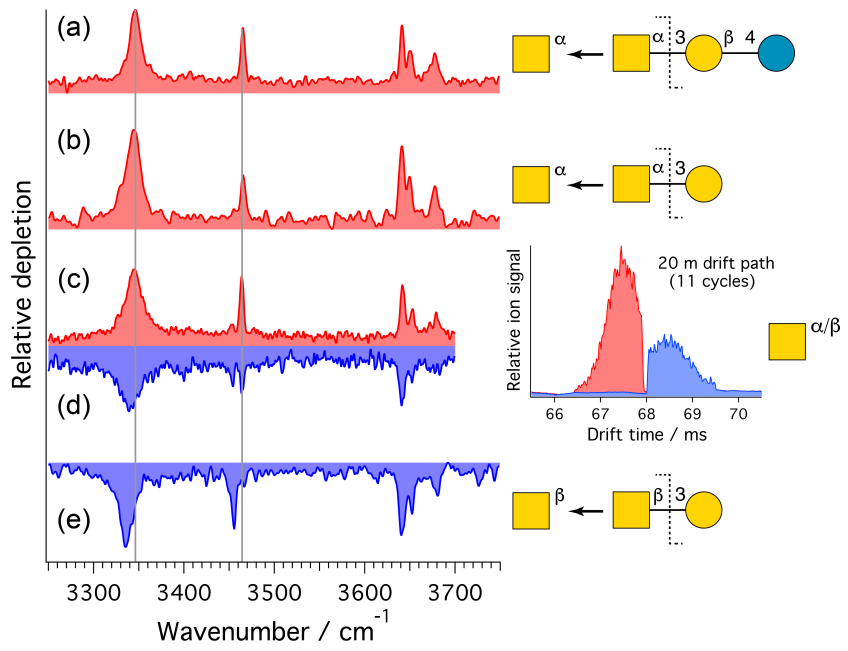

Figure 2: Cryogenic vibrational spectra of $\mathrm{C}_{1}$ fragments of glycans with GalNAc at the non-reducing end. Inset: Highresolution arrival time distribution of GalNAc.

Although we have not assigned the two ion mobility peaks (inset, Fig. 2(c/d)) to the $\alpha$ and $\beta$ anomers, we have shown previously that for small sodiated saccharides structurally similar to those presented in this work, the two mobility peaks observed by ultrahigh-resolution IMS separation correspond to the $\alpha$ and $\beta$ anomers. ${ }^{32}$ 
Furthermore, the fact that the spectrum of the $\mathrm{C}_{1}$ fragment generated at an $\alpha$ glycosidic linkage corresponds exclusively to the spectrum from the first mobility peak, and likewise for the $\beta$ fragment and second mobility peak, provides strong evidence that the two mobility peaks observed correspond to the $\alpha$ and $\beta$ anomers. If mutarotation were to occur upon fragmentation, one would expect a mixture of anomers in the fragment spectra, which is not observed here. There is thus strong evidence that the presence of the $\mathrm{N}$-acetyl group does not affect anomeric retention upon dissociation to form a $\mathrm{C}_{1}$ fragment.

In order to evaluate the generality of anomeric retention further, analogous experiments were carried out on the $\mathrm{C}_{2}$ fragments of the human milk oligosaccharides LNnT and LNnH. In Figure 3 we show the cryogenic vibrational spectra of the $\mathrm{C}_{2}$ fragment from these species and compare them to the spectra of the $\alpha$ and $\beta$ anomers of Galß(1-4)GlcNAc (Figs. 3(a, b)), which we separately measured after separation by SLIM-based ion mobility. ${ }^{33}$ The spectra of the $\mathrm{C}_{2}$ fragments from both LNnT (Fig. 3(c)) and LNnH (Fig. 3(d)) show a good match with the spectrum of the slower mobility peak of Gal $\beta(1-4)$ GlcNAc (Fig. 3(b)). This indicates that $C_{2}$ fragments of the linear tetrasaccharide $\mathrm{LNnT}$ and the branched hexasaccharide LNnH also retain the anomericity of the glycosidic bond.

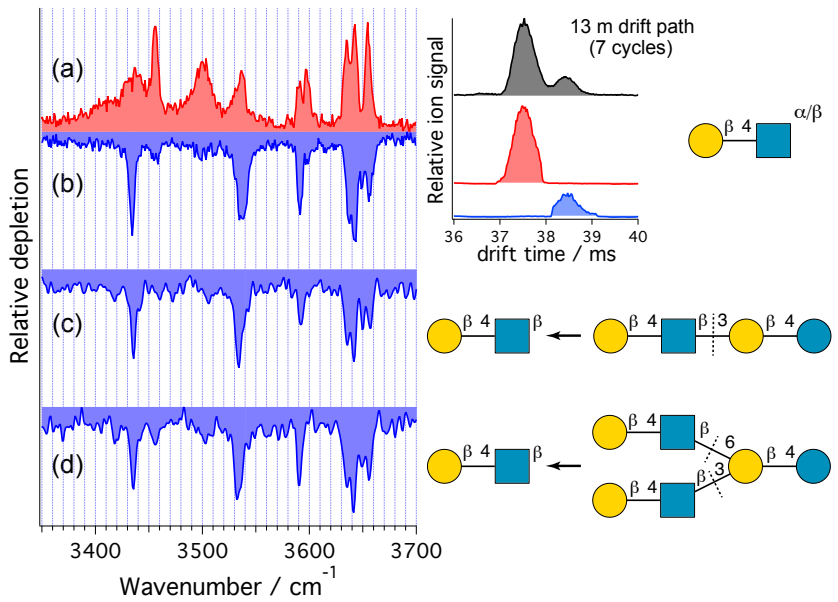

Figure 3: Cryogenic vibrational spectra of the $\mathrm{C}_{2}$ fragments from $\mathrm{LNnT}$ (c) and $\mathrm{LNnH}$ (d) compared to those from the separated anomers of Gal $\beta(1-4) \operatorname{GlcNAc}(a, b))$. Inset: highresolution arrival time distribution of Gal $\beta(1-4)$ GlcNAc. ${ }^{33}$

Our results extend the findings by Compagnon and coworkers ${ }^{4}$ in a significant way. The higher spectroscopic resolution afforded by cryogenic IR spectroscopy together with ultrahigh-resolution ion mobility provides us with the capability of identifying the anomeric forms of larger CID fragments by comparing them to anomerically pure reference compounds. Our observation of anomer retention in the fragmentation of glycans as large as hexasaccharides (Fig. 3(d)), and the fact that it occurs for fragments larger than $C_{1}$ (Figs. 3(c) and 3(d)), suggest that it may be the rule rather than the exception, arising from the large barriers for mutarotation in the gas phase.
If further studies confirm this generality, it would open new possibilities for determination of the primary structures of biologically relevant glycans and provide an important new tool for glycomics.

\section{AUTHOR INFORMATION}

\section{Corresponding Author}

*Email: thomas.rizzo@epfl.ch

ORCID

Eduardo Carrascosa 0000-0003-4338-8669

Lei Yue: 0000-0002-0766-6572

Stephan Warnke: 0000-0001-7481-286X

Thomas R. Rizzo: 0000-0003-2796-905X

\section{ACKNOWLEDGMENT}

The authors thank the Swiss National Science Foundation through grant 200020_184838, the European Research Council (Grant 788697-GLYCANAL), and the EPFL for the financial support of this work.

The authors declare no competing financial interest.

\section{REFERENCES}

1. Rudd, P. M.; Elliott, T.; Cresswell, P.; Wilson, I. A.; Dwek, R. A., Glycosylation and the immune system. Science 2001, 291 (5512), 2370-2376.

2. Stanley, P.; Cummings, R., Structures Common to Different Glycans. In Essentials of Glycobiology, Varki, A.; Cummings, R. D.; Esko, J. D.; Stanley, P.; Hart, G. W.; Aebi, M.; Darvill, A. G.; Kinoshita, T.; Packer, N. H.; Prestegard, J. H.; Schnaar, R. L.; Seeberger, P. H., Eds. Cold Spring Harbor Laboratory Press: Cold Spring Harbor (NY), 2017.

3. Varki, A., Biological roles of glycans. Glycobiology 2016, 27 (1), 3-49.

4. Schindler, B.; Barnes, L.; Renois, G.; Gray, C.; Chambert, S.; Fort, S.; Flitsch, S.; Loison, C.; Allouche, A.-R.; Compagnon, I., Anomeric memory of the glycosidic bond upon fragmentation and its consequences for carbohydrate sequencing. Nat. Comm. 2017, $8(1), 973$

5. Gray, C. J.; Migas, L. G.; Barran, P. E.; Pagel, K.; Seeberger, P. H.; Eyers, C. E.; Boons, G.-J.; Pohl, N. L. B.; Compagnon, I.; Widmalm, G.; Flitsch, S. L., Advancing Solutions to the Carbohydrate Sequencing Challenge. J. Am. Chem. Soc. 2019, 141, 14463-14479.

6. Gray, C. J.; Schindler, B.; Migas, L. G.; Pičmanová, M.; Allouche, A. R.; Green, A. P.; Mandal, S.; Motawia, M. S.; Sánchez-Pérez, R.; Bjarnholt, N.; Møller, B. L.; Rijs, A. M.; Barran, P. E.; Compagnon, I.; Eyers, C. E.; Flitsch, S. L., Bottom-Up Elucidation of Glycosidic Bond Stereochemistry. Anal. Chem. 2017, 89 (8), 4540-4549.

7. Welply, J. K., Sequencing methods for carbohydrates and their biological applications. Trends Biotechnol. 1989, 7 (1), 5-10.

8. Royle, L.; Mattu, T. S.; Hart, E.; Langridge, J. I.; Merry, A. H.; Murphy, N.; Harvey, D. J.; Dwek, R. A.; Rudd, P. M., An analytical and structural database provides a strategy for sequencing O-glycans from microgram quantities of glycoproteins. Anal. Biochem. 2002, 304 (1), 70-90.

9. Mariño, K.; Bones, J.; Kattla, J. J.; Rudd, P. M., A systematic approach to protein glycosylation analysis: a path through the maze. Nat. Chem. Biol. 2010, 6 (10), 713.

10. Harvey, D. J.; Royle, L.; Radcliffe, C. M.; Rudd, P. M.; Dwek, R. A., Structural and quantitative analysis of $\mathrm{N}$-linked glycans by matrix-assisted laser desorption ionization and negative ion 
nanospray mass spectrometry. Anal. Biochem. 2008, 376 (1), 4460.

11. Vliegenthart, J. F.; Dorland, L.; van Halbeek, H., High-resolution, $1 \mathrm{H}$-nuclear magnetic resonance spectroscopy as a tool in the structural analysis of carbohydrates related to glycoproteins. In Advances in Carbohydrate Chemistry and Biochemistry, Elsevier: Amsterdam, 1983; Vol. 41, pp 209-374.

12. Wormald, M. R.; Petrescu, A. J.; Pao, Y.-L.; Glithero, A.; Elliott, T.; Dwek, R. A., Conformational studies of oligosaccharides and glycopeptides: complementarity of NMR, X-ray crystallography, and molecular modelling. Chem. Rev. 2002, 102 (2), 371-386.

13. Harvey, D. J., Collision-induced fragmentation of underivatized Nlinked carbohydrates ionized by electrospray. J. Mass Spectrom. 2000, 35 (10), 1178-1190.

14. Morelle, W.; Michalski, J.-C., Analysis of protein glycosylation by mass spectrometry. Nat. Protoc. 2007, 2 (7), 1585.

15. Gaucher, S. P.; Leary, J. A., Determining anomericity of the glycosidic bond in $\mathrm{Zn}$ (II)-diethylenetriamine-disaccharide complexes using MS $\mathrm{n}$ in a quadrupole ion trap. J. Am. Soc. Mass Spectrom. 1999, 10 (3), 269-272.

16. Harvey, D. J., Identification of protein-bound carbohydrates by mass spectrometry. Proteomics 2001, 1 (2), 311-328.

17. Hsu, H. C.; Liew, C. Y.; Huang, S.-P.; Tsai, S.-T.; Ni, C.-K., Simple method for de novo structural determination of underivatised glucose oligosaccharides. Sci. Rep.-UK 2018, 8 (1), 5562 .

18. Hsu, H. C.; Liew, C. Y.; Huang, S.-P.; Tsai, S.-T.; Ni, C.-K., Simple approach for de novo structural identification of mannose trisaccharides. J. Am. Soc. Mass Spectrom. 2018, 29 (3), 470-480.

19. Ni, C.-K.; Tsai, S.-T.; Liew, C. Y.; Hsu, H. C.; Huang, S.-P.; Weng, W.-C.; Kuo, Y.-H., Automatically Full Glycan Structural Determination with Logically Derived Sequence Tandem Mass Spectrometry. ChemBioChem 2019, 20, 2351-2359.

20. Tsai, S. T.; Liew, C. Y.; Hsu, C.; Huang, S. P.; Weng, W. C.; Kuo, Y. H.; Ni, C. K., Automatic Full Glycan Structural Determination through Logically Derived Sequence Tandem Mass Spectrometry. ChemBioChem 2019, 20, 2351-2359.

21. De Leoz, M. L. A.; Simon-Manso, Y.; Woods, R. J.; Stein, S. E., Cross-Ring Fragmentation Patterns in the Tandem Mass Spectra of Underivatized Sialylated Oligosaccharides and Their Special Suitability for Spectrum Library Searching. J. Am. Soc. Mass Spectrom. 2019, 30 (3), 426-438.

22. Han, L.; Costello, C. E., Mass spectrometry of glycans. Biochemistry-Moscow 2013, 78 (7), 710-720.

23. Domon, B.; Costello, C. E., A systematic nomenclature for carbohydrate fragmentations in FAB-MS/MS spectra of glycoconjugates. Glycoconjugate journal 1988, 5 (4), 397-409.

24. Mucha, E.; González Flórez, A. I.; Marianski, M.; Thomas, D. A.; Hoffmann, W.; Struwe, W. B.; Hahm, H. S.; Gewinner, S.; Schöllkopf, W.; Seeberger, P. H.; von Helden, G.; Pagel, K., Glycan Fingerprinting via Cold-Ion Infrared Spectroscopy. Angew. Chem. Int. Ed. 2017, 56 (37), 11248-11251.

25. Masellis, C.; Khanal, N.; Kamrath, M. Z.; Clemmer, D. E.; Rizzo, T. R., Cryogenic vibrational spectroscopy provides unique fingerprints for glycan identification. J. Am. Soc. Mass Spectrom. 2017, 28 (10), 2217-2222.

26. Khanal, N.; Masellis, C.; Kamrath, M. Z.; Clemmer, D. E.; Rizzo, T. R., Cryogenic IR spectroscopy combined with ion mobility spectrometry for the analysis of human milk oligosaccharides. Analyst 2018, 143 (8), 1846-1852.

27. Rizzo, T. R.; Boyarkin, O. V., Cryogenic Methods for the Spectroscopy of Large, Biomolecular Ions. In Gas-Phase IR Spectroscopy and Structure of Biological Molecules, Rijs, A. M.; Oomens, J., Eds. Springer International Publishing: Switzerland, 2015; pp 43-97.

28. Svendsen, A.; Lorenz, U. J.; Boyarkin, O. V.; Rizzo, T. R., A new tandem mass spectrometer for photofragment spectroscopy of cold, gas-phase molecular ions. Rev. Sci. Instrum. 2010, 81 (7), 073107.

29. Deng, L.; Ibrahim, Y. M.; Hamid, A. M.; Garimella, S. V. B.; Webb, I. K.; Zheng, X.; Prost, S. A.; Sandoval, J. A.; Norheim, R. V.; Anderson, G. A.; Tolmachev, A. V.; Baker, E. S.; Smith, R. D.,
Ultra-High Resolution Ion Mobility Separations Utilizing Traveling Waves in a $13 \mathrm{~m}$ Serpentine Path Length Structures for Lossless Ion Manipulations Module. Anal. Chem. 2016, 88 (18), 8957-8964.

30. Webb, I. K.; Garimella, S. V. B.; Norheim, R. V.; Baker, E. S.; Ibrahim, Y. M.; Smith, R. D., A Structures for Lossless Ion Manipulations (SLIM) Module for Collision Induced Dissociation. J. Am. Soc. Mass Spectrom. 2016, 27 (7), 1285-1288.

31. Ben Faleh, A.; Warnke, S.; Rizzo, T. R., Combining UltrahighResolution Ion-Mobility Spectrometry with Cryogenic Infrared Spectroscopy for the Analysis of Glycan Mixtures. Anal. Chem. 2019, 91 (7), 4876-4882.

32. Warnke, S.; Ben Faleh, A.; Scutelnic, V.; Rizzo, T. R., Separation and Identification of Glycan Anomers Using Ultrahigh-Resolution Ion Mobility Spectrometry and Cryogenic Ion Spectroscopy. $J$. Am. Soc. Mass Spectrom. 2019, 30 (11), 2204-2211.

33. Warnke, S.; Faleh, A. B.; Pellegrinelli, R. P.; Yalovenko, N.; Rizzo, T. R., Combining ultra-high resolution ion mobility spectrometry with cryogenic IR spectroscopy for the study of biomolecular ions. Faraday Discuss. 2019, 217, 114. 
For Table of Contents Only:

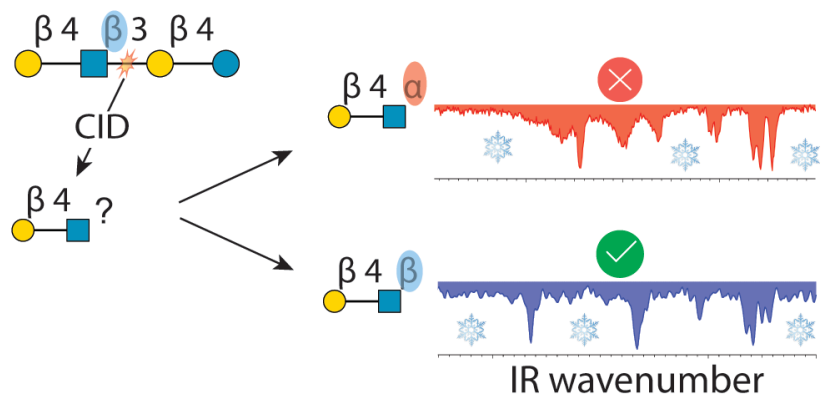

